\title{
Trombosis venosa profunda de los miembros inferiores: utilidad de la ecografía doppler
}

\author{
Doppler ecography: a safe method for diagnosis of deep \\ venous thrombosis
}

Carlos Alberto Correa-Rodríguez MD. ${ }^{1}$

\begin{abstract}
In this review, the different diagnostic methods of deep vein thrombosis will be treated, to which the first symptoms of the pathology appear once, and as it has varied until currently using Doppler Ultrasound, which provides a safe noninvasive method able to provide an anatomical and hemodynamic topography.

\section{RESUMEN}

En esta revisión, se tratarán los diferentes métodos de diagnóstico de la trombosis venosa profunda, a los que se recurren una vez los primeros síntomas de la patología aparece, y como ha variado hasta usar actualmente la ecografía doppler, que proporciona un método seguro no invasivo capaz de suministrar una topografía anatómica y hemodinámica.
\end{abstract}

\section{Key words:}

Doppler ultrasound, lower limbs, deep vein thrombosis

\section{Palabras clave:} Ecografía doppler, miembros inferiores, trombosis venosa profunda a trombosis venosa profunda (TVP) es una patología común sobre todo en personas de edad avanzada y/o pacientes hospitalizados. Es una enfermedad frecuente pues se estima que la incidencia media en la población es de 5 por 10.000 habitantes cada año y esto aumenta significativamente con la edad. Para personas de 30 a 49 años es de aproximadamente 2-3/10.000 y para personas de 7079[1]. 20/10.000. Aproximadamente el 1\%-4\% de todas las TVP involucran las extremidades superiores, todavía hay sustancialmente menos información disponible sobre la epidemiología, incidencia real, manejo y pronóstico en la extremidad superior de TVP en la población general[2]. Por otra parte, se estima que la incidencia de trombosis venosa profunda en los miembros inferiores se encuentra entre 40 a 182 por 100.000 habitantes[3]. La incidencia de TVP en la población mundial aumenta con la edad en $1 \%$ y con los factores de riesgo asociados tales como hospitalizaciones, cáncer, tabaquismo, anticonceptivos orales,

Fecha de recepción: 06 de noviembre de 2019

Fecha de aceptación: 19 de noviembre de 2019

\section{ORCID}

https://orcid.org/0000-0003-0357-5101

Correspondencia

Carlos Alberto Correa-Rodríguez

Email.carlos.correa.rodriguez@correounivalle.edu.co 
hipertensión y diabetes[4].

El tratamiento se basa principalmente en la terapia anticoagulante, lo que añade el riesgo de hemorragia. Estos factores hacen que el diagnóstico preciso y temprano de la enfermedad resulte crucial. El tromboembolismo venoso recurrente generalmente se sospecha después de la suspensión de anticoagulantes, cuyos síntomas y signos clínicos pueden confundirse con los del síndrome post-trombótico, como el dolor y el edema, que representan el $25-50 \%$ de los pacientes a los 2 años de tratamiento[5]. Junto con el potencial de una duración indefinida de la terapia anticoagulante si se confirma la recurrencia, además, del riesgo de complicaciones hemorrágicas con el tratamiento prolongado de anticoagulación, esto resalta la necesidad de técnicas precisas para diagnosticar la recurrencia de la TVP[6].

Debido a que la TVP es una enfermedad más frecuente de lo que se creía, se hace necesario entonces, tener diferentes métodos de diagnóstico, que permitan al personal de salud realizar una intervención clínica lo más pronto posible una vez que los primeros síntomas de la patología aparezcan. El diagnóstico de la TVP es a menudo difícil y se basa, en primer lugar, en la ecografía venosa. Tanto en la medicina de emergencia como la literatura sobre cuidados críticos han demostrado que, después de una breve capacitación enfocada, los médicos y otros profesionales de la salud pueden realizar una ecografía de compresión venosa rápidamente con una alta precisión diagnóstica para la TVP de las extremidades inferiores[7,8].

Se han estudiado varios métodos para diagnosticar la TVP; la venografía de contraste se considera como el "Gold standard" en el diagnóstico de la TVP, con una sensibilidad del 99\%. La venografía de contraste, sin embargo, rara vez se utiliza, debido a que es costosa de realizar y requiere de una unidad de radiología especializada para realizar el examen. Hasta un $2 \%$ de los pacientes sometidos a este procedimiento han presentado complicaciones que incluyen TVP post-inyección debido al colorante de contraste, algunos han presentado extravasiones, fleblitis superficial y reacciones alérgicas[9]. La ventaja de la venografía es su detección precisa (100\%) y directa de la pantorrilla.

Existen también otros métodos de diagnóstico, como por ejemplo la pletismografía de impedencia, una técnica que no es invasiva pero que presenta sensibilidad inadecuada haciendo que el diagnóstico de TVP sea difícil; por su parte, la imagen de resonancia magnética tiene una alta precisión, pero es no se puede usar en mujeres embarazadas y es una técnica costosa y no se encuentra disponible en hospitales de un nivel bajo. Al igual que la resonancia magnética, la tomografía computarizada es una técnica no invasiva que puede ayudar a diagnosticar una TVP pélvica, también puede excluir simultáneamente embolia pulmonar, pero al igual que la resonancia magnética es una técnica muy costosa y sus datos son limitados. La ultrasonografía es una técnica que provee una alta precisión en el diagnóstico y no es invasiva, sin embargo, nos es preciso usarla para la TVP de la pantorrilla o de pelvis y para realizar un estudio completo de la TVP se requiere mucho tiempo.

En contraste, la ecografía doppler es el único procedimiento no invasivo capaz de suministrar una topografía anatómica y hemodinámica para la circulación venosa de las extremidades inferiores en tiempo real, por lo que constituye el método diagnóstico de elección[10]. Los estudios vasculares tradicionales, los exámenes "dúplex" y "triplex", utilizan una combinación de imágenes bidimensionales (2-D) con compresión junto con el uso de color y/o doppler espectral. Sin embargo, si se usan juntos correctamente, la ecografía de compresión y la ecografía doppler color o espectral como métodos complementarias se consideran que ambas técnicas proporcionan los medios más precisos para el diagnóstico de la trombosis venosa profunda aguda[11].

El ultrasonido Doppler es el método de elección para evaluar la insuficiencia venosa, su fisiopatología y diagnóstico. Un conocimiento integral de la anatomía, así como de las posibles alteraciones funcionales detectadas por el estudio Doppler, son cruciales para la evaluación de la TVP y la confección de un "mapa ecográfico que permita a los médicos o cirujanos vasculares el tratamiento más adecuado para cada paciente[9]. Estudios más recientes han demostrado que los exámenes de ecografía con compresión 2-D solo producen una precisión similar a la de los estudios dúplex tradicionales o triplex vasculares[8],[12]-[14]. La notificación de los exámenes dúplex o triplex a menudo se retrasa debido a limitaciones en la disponibilidad de los servicios de radiología. Los retrasos resultantes en la obtención de dichos resultados de las pruebas pueden comprometer la atención de los pacientes con una enfermedad aguda.

La ecografía dúplex de la extremidad inferior tiene una serie de ventajas sobre la venografía convencional en la evaluación de la TVP aguda. Primero, la ecografía es más rápida, fácil y portátil de realizar en todo tipo de pacientes en el hospital. En segundo lugar, este tipo de ecografía no utiliza radiación ionizante y no expone a los pacientes a ninguno de los riesgos asociados con el material de contraste yodado, además, puede ayudar a mostrar otras causas de síntomas de las extremidades inferiores, como quistes 
poplíteos o pseudoaneurismas, que pueden no ser evidentes en la venografía convencional[11].

Existen dos tipos de Doppler para realizar la ecografía del sistema venoso tanto de miembros inferiores como de miembros superiores. El primero el Doppler de flujo de color se puede usar para ayudar a distinguir los vasos de las estructuras circundantes. El color será asimétrico en el lumen de las venas con trombosis aguda y crónica, llenando solo la porción de lumen sin coágulo, pero los defectos de "relleno" Doppler de color no se deben usar solo para determinar la presencia o ausencia de un coágulo. La turbulencia puede generar el artefacto "ruido" pudiéndose observar en áreas estenóticas[15].

Por su parte el Doppler espectral permite una evaluación de las características y la velocidad del flujo sanguíneo[16]. Las muestras se obtienen colocando la caja del Doppler de onda pulsada sobre la vena en un plano longitudinal con un ángulo de insonación mantenido a menos de 60 grados[11]. La función Doppler revela una visualización gráfica del flujo sanguíneo. La espontaneidad se refiere a la presencia o ausencia de flujo venoso. El flujo sanguíneo normal medido por eco doppler es bifásico o pico. La velocidad normal del flujo sanguíneo venoso varía entre 15 y 20 cm[17].

Una vez más, si hay dificultad para diferenciar la arteria de la vena, se puede usar Doppler color para determinar el flujo, y la intensidad del flujo descendente será evidente como una representación gráfica. Para el persona de salud que están familiarizados con el uso del Doppler espectral, las formas de onda arterial y venosa se pueden evaluar con un flujo venoso que aparecerá como un patrón ondulante más lento en comparación con el flujo arterial trifásico que se escucha tradicionalmente durante el Doppler US de los miembros inferiores o superiores[18].

En conclusión, la trombosis venosa profunda es una patología común en los pacientes hospitalizados, con una incidencia que aumenta a medida que se incrementa la edad del paciente. El manejo intrahospitalario de estos pacientes puede generar complicaciones y su diagnóstico oportuno puede estar a la mano de los médicos, tanto de urgencias como de los hospitalarios a través de la ecografía Doppler que es un método no invasivo, fácil de manejar y accesible para ser usado en los diferentes centros médicos.

\section{Referencias}

1. Fowkes FJ, Price JF, Fowkes FG. Incidence of diagnosed deep vein thrombosis in the general population: systematic review. Eur J Vasc Endovasc Surg. 2003 Jan;25(1):1-5. https://doi. org/10.1053/ejvs.2002.1778 PMID:12525804

2. Isma N, Svensson PJ, Gottsäter A, Lindblad B. Upper extremity deep venous thrombosis in the population-based Malmö thrombophilia study (MATS). Epidemiology, risk factors, recurrence risk, and mortality. Thromb Res. 2010 Jun;125(6):e335-8. https://doi.org/10.1016/j. thromres.2010.03.005 PMID:20406709

3. Li W, Chuanlin Z, Shaoyu M, Yeh $\mathrm{CH}$, Liqun C, Zeju Z. Catheterdirected thrombolysis for patients with acute lower extremity deep vein thrombosis: a meta- analysis. Rev Lat Am Enfermagem. 2018 Jun;26(0):e2990. https://doi.org/10.1590/15188345.2309.2990 PMID:29947719

4. Ortiz P, Javier JJ, Carvalho R. Patología del sistema venoso profundo. Enfermedad, trombo embolica-ETE. Revisión del tema, pautas de tratamientos y profilaxis. Biomedicina. 2013;8(3):2436.

5. Prandoni $\mathbf{P}$. The long term clinical course of acute deep vein thrombosis. 1996; (May 2014).

6. Maufus M, Elias A, Barrellier MT, Pernod G; French Society for Vascular Medicine. Diagnosis of deep vein thrombosis recurrence: ultrasound criteria. Thromb Res. 2018 Jan; 161(161):7883. https://doi.org/10.1016/j. thromres.2017.11.004 PMID:29216479

7. Blaivas M, Lambert MJ, Harwood RA, Wood JP, Konicki J. Lower- extremity Doppler for deep venous thrombosis-can emergency physicians be accurate and fast? Acad Emerg Med. 2000 Feb;7(2):120-6. https://doi. org/10.1111/j.1553-2712.2000. tb00512.x PMID:10691069

8. Magazzini S, Vanni S, Toccafondi S, Paladini B, Zanobetti M, Giannazzo G, et al. Duplex ultrasound in the emergency department for the diagnostic management of clinically suspected deep vein thrombosis. Acad Emerg Med. 2007 Mar;14(3):216-20. https://doi. org/10.1197/j.aem.2006.08.023 PMID:17264203

9. Berardi $\mathrm{H}, \mathrm{Ciccioli} A$. Examen Doppler de la insuficiencia venosa de miembros inferiores: consenso entre especialistas. Rev Argent Radiol. 2015;79(2):729. https://doi.org/10.1016/j. rard.2014.10.008.

10. García Carriazo M, Gómez De 
Las Heras C, Mármol Vázquez $\mathrm{P}$, Ramos Solís MF. Estudio de la insuficiencia venosa crónica mediante ecografía Doppler y realización de cartografía venosa. Radiologia (Madr). 2016;58(1):7-15. https://doi. org/10.1016/j.rx.2015.10.006 PMID:26655801

11. Maki DD, Kumar N, Nguyen B, Langer JE, Miller WT Jr, Gefter WB. Distribution of thrombi in acute lower extremity deep venous thrombosis: implications for sonography and CT and MR venography. AJR Am J Roentgenol. 2000 Nov;175(5):1299301. https://doi.org/10.2214/ ajr.175.5.1751299 PMID:11044027

12. Bernardi E, Camporese G. Diagnosis of deep-vein thrombosis. Thromb Res.
2017 Sep;163(163):201-6. https://doi.org/10.1016/j. thromres.2017.10.006 PMID:29050648

13. Lichtenstein D. FALLS-protocol: lung ultrasound in hemodynamic assessment of shock. Heart Lung Vessel. 2013;5(3):142-7. PMID:24364005

14. Chin EE, Zimmerman PT, Grant EG. Sonographic evaluation of upper extremity deep venous thrombosis. J Ultrasound Med. 2005 Jun;24(6):82938. Available from: http:// www.ncbi.nlm.nih.gov/pubmed/15914687 https://doi. org/10.7863/jum.2005.24.6.829 PMID:15914687

15. Knudson GJ, Wiedmeyer DA, Erickson SJ, Foley WD, Lawson $\mathrm{TL}$, Mewissen MW, et al. Color Doppler sonographic imaging in the assessment of upperextremity deep venous thrombosis. AJR Am J Roentgenol. 1990 Feb;154(2):399-403. https://doi. org/10.2214/ajr.154.2.2136963 PMID:2136963

16. Nilam J. Soni RA\& PK. Point-ofCare Ultrasound. (ELSEVIER, ed.). Philadelphia doi:10.1016/B9781-4557-7569-9.00008-6

17. Coffman JD, Lempert JA. Venous flow velocity, venous volume and arterial blood flow. Circulation. 1975 Jul;52(1):141-5. https://doi. org/10.1161/01.CIR.52.1.141 PMID:1132117

18. Tracy JA, Edlow JA. Ultrasound diagnosis of deep venous thrombosis. Emerg Med Clin North Am. 2004 Aug;22(3):775-96. https://doi. org/10.1016/j.emc.2004.04.008 PMID:15301850 